\title{
CAR T-cell approvals: multiple myeloma likely next up
}

\author{
Sharon Worcester
}

The next major approval in the chimeric antigen receptor (CAR) T-cell therapy arena will target multiple myeloma, according to Carl June, MD, the Richard W Vague Professor in Immunotherapy and a pioneer in CAR T-cell research at the University of Pennsylvania, Philadelphia. That approval is anticipated sometime in 2019, and will "completely transform oncology,"

Dr June said in a recent interview. "Myeloma is the most common blood cancer in adults, and there's never been a curative therapy, but now there is a subset of patients who look like they're cured with CAR T cells."

\section{Researcher-turned-patient}

The first treated patient in a trial of a novel anti-B-cell maturation antigen (BCMA)-specific CAR T-cell therapy $(\text { CART-BCMA })^{1}$ developed by University of Pennsylvania researchers in collaboration with Novartis is part of that subset. Earlier this year, Woodring Wright, MD, a profes-
Dr Carl June

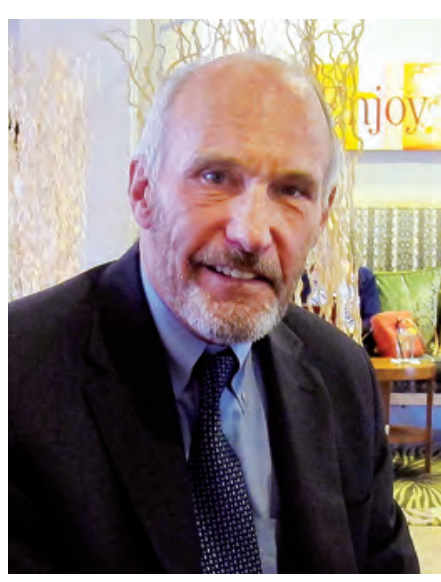
sor of cell biology and medicine at the University of Texas (UT) Southwestern Medical Center in Dallas, outed himself as that first patient when he announced that CART-BCMA saved his life. ${ }^{2}$

Dr Wright had been diagnosed with multiple myeloma about 12 years ago and had failed 11 previous chemotherapies before he was enrolled in the CART-BCMA trial. He remains cancer free more than 2 years after receiving CART-BCMA and he's now conducting CAR T-cell-related research in his UT Southwestern laboratory to broaden the effectiveness of current CAR T-cell therapies. In particular, he is looking at whether the small percentage of patients in whom CAR T-cell therapy does not work might benefit from telomerase to lengthen telomeres, because most patients who fail CAR T-cell therapy are elderly and might have terminally short telomeres. ${ }^{2}$

\section{Pharma lines up the trials}

An ongoing University of Pennsylvania trial led by Adam D Cohen, MD, director of myeloma immunotherapy at the Abramson Cancer Center, has an overall response rate of 64\%; initial phase 1 efficacy and safety results were reported at the 2016 annual meeting of the American Society of Hematology (ASH). ${ }^{3}$ In addition, multiple companies are pursuing registration trials for CAR T-cell therapies in myeloma, Dr June said.

Among those companies are bluebird bio and Celgene, which together are developing an antiBCMA CAR T-cell therapy known as bb2121. The product was granted breakthrough therapy designation by the US Food and Drug Administration in November 2017 and will thus receive expedited review by the agency. It has also been fast-tracked in Europe.

The decision to fast-track bb2121 in the United States was based on preliminary results from the CRB410 trial. ${ }^{4}$ Updated findings from that trial were presented at the 2017 ASH annual meeting and showed an overall response rate of $94 \%$ in 21 patients, with 17 of 18 patients who received doses above $50 \times 10^{6} \mathrm{CAR}+\mathrm{T}$ cells having an overall response, and 10 of the 18 achieving complete remission. The progression-free survival rates were $81 \%$ at 6 months, and $71 \%$ at 9 months, with responses deepening over time. The complete response rates were $27 \%$ and $56 \%$ in May and October of 2017, respectively.

Responses were durable, lasting more than 1 year in several patients, the investigators reported. Phase 2 of the trial - the global pivotal KarMMA trial - is currently enrolling and will dose patients at between 150 and $350 \times 10^{6} \mathrm{CAR}+\mathrm{T}$ cells. ${ }^{5}$

Janssen Biotech Inc and Legend Biotech USA Inc/ Legend Biotech Ireland Ltd have also joined forces to develop an anti-BCMA CAR T-cell 
product for multiple myeloma, Dr June said. The companies announced in late 2017 that they had entered into "a worldwide collaboration and license agreement" to develop the CAR T-cell drug candidate, LCAR-B38M. ${ }^{6}$ It has been accepted for review by the China Food and Drug Administration and is in the planning phase of clinical studies in the United States for multiple myeloma, according to that announcement.

Cost, financial toxicity, and a new therapeutic landscape The rush for the approval of a CAR T-cell therapy for myeloma will lead to a welcome addition to the treatment armamentarium not just because of the clinical benefits, but because of the possibility of reducing disease-related costs (p. e177). Although myeloma represents only about $2 \%$ of all cancers, it is responsible for $7 \%$ of cancer costs, $\mathrm{Dr}$ June noted, and since many patients live with their disease for a long time, that can mean substantial "financial toxicity" being associated with treatment for the disease. "So CAR T-cell therapy for myeloma will bring a huge change to the practice of oncology," he added.

Dr June explained that tisagenlecleucel, the first CAR T-cell therapy to be approved (in August 2017; p. e126), was for pediatric acute lymphoblastic leukemia that had relapsed at least twice. " "That's only about 600 kids a year in the United States, so it's an ultra-orphan market," he said. However, with the subsequent October 2017 approval of axicabtagene ciloleucel for certain cases of large B-cell lymphoma ${ }^{8}$ and the anticipated myeloma approval, CAR T-cell therapy will move away from that orphan status.

"There are a lot of difficulties whenever you change to something new," he said, comparing the CAR T-cell therapy evolution to that of bone marrow transplantation in the 1980s, when many voiced concern about the new ther-

\section{References}

1. University of Pennsylvania. CART-BCMA cells for multiple myeloma. https://clinicaltrials.gov/ct2/show/NCT02546167. NCT02546167. Accessed June 13, 2018.

2. Frisinger C. Cancer researcher's life saved by CAR-T treatment. UT Southwestern Medical Center website. https://www.utsouthwestern.edu/newsroom/articles/year-2018/wright-car-t.html. Published. Accessed June 13, 2018.

3. Cohen AD, Garfall AL, Stadtmauer EA, et al. B-cell maturation antigen (BCMA)-specific chimeric antigen receptor T cells (CARTBCMA) for multiple myeloma (MM): initial safety and efficacy from a phase I study. Blood. 2016;128(22):1147.

4. Berdeja JG, Lin Y, Raje N, et al. Durable clinical responses in heavily pretreated patients with relapsed/refractory multiple myeloma: updated results from a multicenter study of bb2121 anti-BCMA CAR T cell therapy. Blood. 2017;130:740.

5. Celgene. Efficacy and safety study of bb2121 in subjects with relapsed and refractory multiple myeloma (KarMMa) (bb2121). https://clinicaltrials.gov/ct2/show/NCT03361748. NCT03361748. Accessed June 13,2018. apy because it was available at only 2 centers in the United states and required a high level of specialized skill. "But over the years, millions of transplants have been done [and] they're done at many community centers. And it's the same thing with CARs." There are now 30 centers offering CAR T-cell therapy and people have to be trained. "It's a new skill set, and it will take time," he said.

\section{Access to trials: balancing demand and availability}

That delay can be particularly frustrating because there are many patients who might benefit "in a major way" from CAR T-cell therapy, but who can't get on a clinical trial, Dr June noted.

"There's more demand than availability, and it's going to take a while" for that to change, he said. The solution most likely will involve the complementary use of off-the-shelf CAR T cells in some patients to induce remission and perhaps provide a bridge to another definitive therapy, and ultrapersonalized CAR T-cell therapy in others, as well as combinations that include CAR T cells and targeted agents or checkpoint inhibitors.

CRISPR-Cas9 gene editing is also being considered as a tool for engineering multiple myeloma cellular immunotherapy (and other cancer treatments), as in the Parker Institute-funded NYCE study, ${ }^{9}$ Dr June said. "We're actually removing the [programmed death-1] gene and the T-cell receptors ... it shows enormous potential for gene editing. CRISPR is going to be used for a lot of things, but the first use is with T-cell therapies, so we're really excited about that trial."

Disclosures. Dr June reported royalties and research funding from Novartis and an ownership interest in Tmunity Therapeutics.

6. Janssen enters worldwide collaboration and license agreement with Chinese company Legend Biotech to develop investigational CAR-T anti-cancer therapy. https://www.jnj.com/media-center/ press-releases/janssen-enters-worldwide-collaboration-and-licenseagreement-with-chinese-company-legend-biotech-to-develop-investigational-car-t-anti-cancer-therapy. New Brunswick, NJ: Johnson \& Johnson. December 21, 2017. Accessed June 13, 2018.

7. FDA approves tisagenlecleucel for B-cell ALL and tocilizumab for cytokine release syndrome. FDA News Release. August 30, 2017. https://www.fda.gov/Drugs/InformationOnDrugs/ApprovedDrugs/ ucm574154.htm. Accessed June 13, 2018.

8. FDA approves axicabtagene ciloleucel for large B-cell lymphoma. FDA News Release. October 18, 2017. https://www.fda.gov/Drugs/ InformationOnDrugs/ApprovedDrugs/ucm581296.htm. Accessed June 13, 2018.

9. University of Pennsylvania. NY-ESO-1-redirected CRISPR (TCRendo and PD1) edited T cells (NYCE T Cells). NCT03399448. Accessed June 13, 2018. 